\title{
Type III secretion systems: jumping into the reservoir
}

Increasing evidence suggests that free-living protozoans, such as amoebae, are important environmental reservoirs for foodborne pathogens, but how the bacteria infect and survive in protozoan hosts is unclear. A recent study published in Applied Environmental Microbiology suggests that type III secretion systems (T3SSs) hold the key.

Salmonella enterica serovars have been shown to survive and proliferate in amoebae. Bleasdale and colleagues investigated whether two S. enterica T3SSs that are known to be involved in survival in mammalian cells have similar roles when these bacteria infect amoebae. These secretion systems are T3SS-1 and T3SS-2, which are encoded by Salmonella pathogenicity island 1 (SPI-1) and SPI-2, respectively. Variants of Salmonella enterica subsp. enterica serovar Typhimurium were used that carried mutations for the SPI-1 gene spaS, the SPI-2 gene ssaU or the phoP gene, which encodes a component of a two-component regulatory system that functions together with T3SS-2 to promote survival in mammalian cells.

The authors monitored the uptake and survival of these S. enterica strains into the amoeba Acanthamoeba polyphaga. The spaS mutant was recovered from lysed amoebal cells at similar levels to a wild-type strain at all time points after infection. By contrast, the ssaU and $p h o P$ mutants were recovered



NEIL SMITH

at significantly lower levels, with a decline over time in the number of viable bacteria. These results suggest that T3SS-2, but not T3SS-1, is required for $S$. enterica survival in amoebae. In support of this conclusion, the authors used realtime RT-PCR (PCR after reverse transcription of RNA) to show that a key SPI-2 gene undergoes a dramatic increase in transcription 4 hours after infection, whereas its SPI-1 counterpart shows only a transient increase.

As well as the implications for understanding how bacterial pathogens infect and survive in protozoan reservoirs, this study raises an interesting evolutionary possibility: did mechanisms that have been considered primarily in terms of virulence with respect to animal hosts in fact originate to allow survival in different organisms?

Louisa Flintoft, Chief Editor, Nature Reviews Genetics

ORIGINAL RESEARCH PAPER Bleasdale, B. et al. The Salmonella pathogenicity island (SPI)-2 -encoded type III secretion system is essential for survival of Salmonella enterica serovar Typhimurium in free-living amoebae. Appl. Environ. Microbiol. 23 Jan 2009 (doi:10.1128/ AEM.02033-08) 